\title{
Empowerment von Frauen in sozial benachteiligten Lebenslagen: Das BIG-Projekt
}

\section{Empowerment of Women in Difficult Life Situations: The BIG Project}

Autoren

Institut
A. Rütten, U. Röger, K. Abu-Omar, A. Frahsa

Institut für Sportwissenschaft und Sport, Universität Erlangen-Nürnberg

\section{Schlüsselwörter \\ - Empowerment \\ - Partizipation \\ - Gesundheitsförderung \\ ○ körperliche Aktivität}

\section{Key words}

- empowerment

- participation

- health promotion

- physical activity

\section{Bibliografie}

DOI $10.1055 / \mathrm{s}-0028-1103262$

Gesundheitswesen 2008;

70: $742-747$

(c) Georg Thieme Verlag KG

Stuttgart · New York

ISSN 0941-3790

Korrespondenzadresse

\section{Prof. Dr. A. Rütten}

Institut für Sportwissenschaft und Sport

Universität Erlangen-Nürnberg

Gebbertstr. 123b

91058 Erlangen

karim@sport.uni-erlangen.de

\section{Zusammenfassung \\ $\nabla$}

BIG ist ein Projekt zur Förderung von Gesundheit über Sport und Bewegung bei Frauen in schwierigen Lebenslagen. Im Sinne der Gesundheitsförderung der WHO sollen die Frauen dabei befähigt werden, die Kontrolle über Determinanten für ihre Gesundheit durch Bewegungsförderung zu erhöhen. Zur Anwendung kam ein umfassender partizipativer Ansatz, über den die Frauen in die Planung, Implementierung und Evaluierung des Projekts einbezogen wurden. Die Auswirkungen von BIG auf das beabsichtigte „Empowerment“ der Frauen wurden in Form von halbstrukturierten qualitativen Interviews mit insgesamt 15 an BIG beteiligten Frauen erhoben. Ausgewertet wurden die Daten mittels qualitativer Inhaltsanalyse. Die Ergebnisse zeigen eine „Befähigung“ der Frauen auf individueller Ebene, so z. B. die Erweiterung von Kompetenz und erfahrener Selbstwirksamkeit in Bezug auf Sport und Gesundheit sowie die Verbesserung von Sozialkontakten. Diese Effekte werden durch Prozesse einer „Bemächtigung" auf organisatorischer und gemeindebezogener Ebene unterstützt, die den Frauen die Übernahme der Kontrolle über ihre Lebenswelt und die sie beeinflussende Politik ermöglichen. Somit stellt der umfassende partizipative Ansatz von BIG einen zentralen Erfolgsfaktor im Hinblick auf die Förderung des Empowerment von Frauen in schwierigen Lebenslagen dar.

\section{Einleitung}

\section{Der Ansatz von BIG}

BIG steht für „Bewegung als Investition in Gesundheit“. Hauptziel von BIG ist die Förderung von Sport und Bewegung bei Frauen in schwierigen Lebenslagen ${ }^{1}$. Hierfür wurden die Settings Betrieb, Sportverein und Wohnquartier gewählt. Die Planung, Implementierung und Evaluierung

\section{Abstract \\ $\nabla$}

BIG is a project for the promotion of physical activity among women in difficult life situations. Following the main health promotion principles of the WHO, the women shall be enabled or empowered to take control of determinants of their health. A comprehensive participatory approach was applied and women were included in planning, implementing and evaluating the project. For measuring the effects of BIG on the empowerment of participating women, qualitative semi-structured interviews with 15 women participating in BIG were conducted. For data analysis, qualitative content analysis was used. Results showed the empowerment of the women on the individual level as they gained different competencies and perceived self-efficacy. These effects were supported through the empowerment process on the organizational and community levels where women gained control over their life situations and over policies influencing them. Therefore, the participatory approach of BIG is a key success factor for empowerment promotion of women in difficult life situations. 


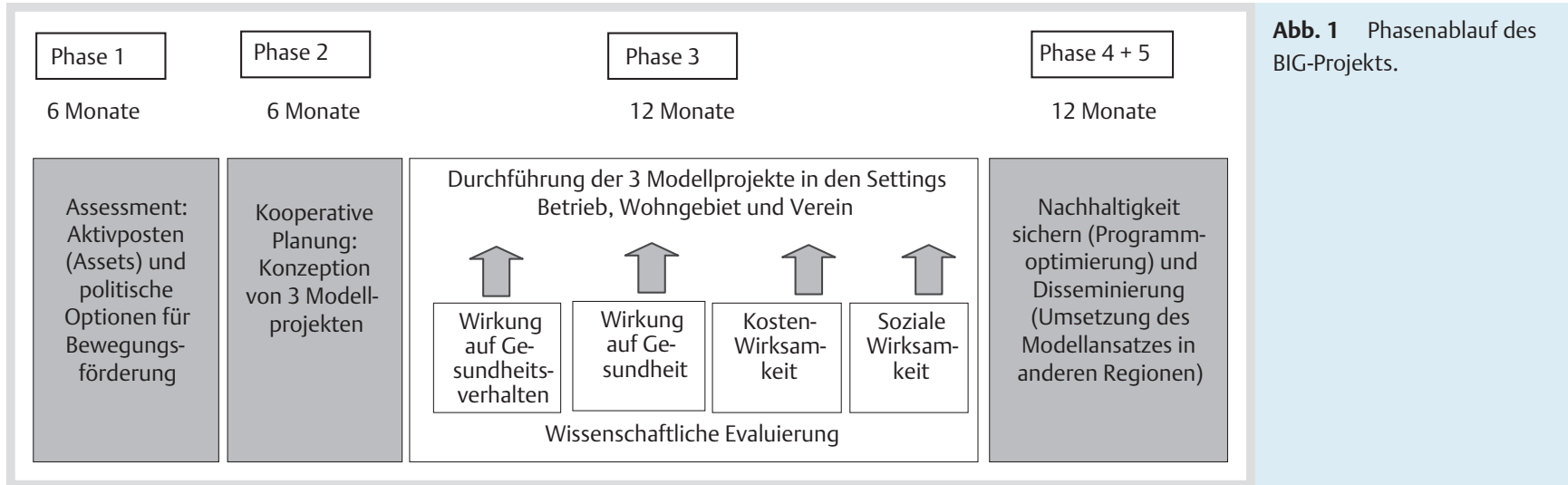

zogen wurden. Dabei ist ein multidimensionales Interventionskonzept für die Bewegungsförderung der Zielgruppe entstanden, das wohnortnahe und kostengünstige Bewegungsprogramme mit Kinderbetreuung, eine Frauenbadezeit, Schwimm- und Aquafitness-Kurse für Frauen, Bauchtanz- und Selbstverteidigungskurse sowie Gesundheitsseminare umfasst. Gleichzeitig wurden Projektbüros eingerichtet, die von den Frauen selbst geführt werden und über die die Projektarbeit organisiert und koordiniert wird.

\section{Evaluation von BIG}

BIG wird durch eine umfassende Evaluation begleitet. Die Evaluation von BIG (vgl. $\odot$ Abb. 1) bezieht sich sowohl auf den gesamten Prozess der Projektentwicklung, -umsetzung und -nachhaltigkeit (z. B. unter dem Aspekt der Beteiligung der Zielgruppe) als auch auf die Projektergebnisse, die mit der multidimensionalen Intervention erzielt wurden. Für die Evaluation der multidimensionalen Intervention kamen verschiedene Methoden zur Anwendung. Zum einen wurde eine standardisierte Längsschnittstudie angelegt, bei der physiologische Tests und eine standardisierte Befragung der Programmteilnehmerinnen zur Erhebung von Veränderungen des Gesundheitszustandes und des Gesundheitsverhaltens sowie der gesundheitsökonomischen Auswirkungen durchgeführt wurden. Darüber hinaus kamen qualitative Methoden der Datenerhebung wie Dokumentenanalyse, Beobachtung und qualitative Interviews zum Einsatz, um Wirkungen auf die individuelle Befähigung sowie die sozialen und politisch-organisatorischen Rahmenbedingungen von BIG zu überprüfen. So wurde in der BIG-Evaluation beispielsweise nachgewiesen, dass Frauen in schwierigen Lebenslagen mit den BIG Bewegungsaktivitäten tatsächlich erreicht (Reach) und Wirkungen auf physiologische Gesundheitsparameter (Blutdrucksenkung und Erhöhung der Herzfrequenzvariabilität sowie Steigerung der körperlichen Aktivierung) erzielt wurden. Darüber hinaus konnten auch politisch-organisatorische Veränderungen wie die Einrichtung einer Planstelle in der Stadtverwaltung erzielt werden.

Der Empowerment-Ansatz in der Gesundheitsförderung „Empowerment“ ist der zentrale Begriff der internationalen Gesundheitsförderungsbewegung. Definiert man Gesundheitsförderung nach der Ottawa Charta (ergänzt durch die Jakarta Erklärung der WHO) [2,3] als „Prozess, der Menschen befähigt, die Kontrolle über die (Determinanten für die) Gesundheit zu erhöhen und (dadurch) ihre Gesundheit zu verbessern“, so wird Empowerment zum Dreh- und Angelpunkt aller Bereiche gesundheitsförderlichen Handelns. Hier wird zugleich die Mehr- dimensionalität des Begriffs Empowerment deutlich, die seine Übersetzung ins Deutsche so schwierig macht: Zum einen geht es um „Befähigung“ im Sinne der Entwicklung individueller Kompetenzen und sozialen Handelns (gesundheitsförderliche Gemeinschaftsaktionen), zum anderen aber auch um „Bemächtigung“, d.h. um die Kontrolle der Menschen über ihre Lebenswelten und über die Politik, die die Gesundheitsförderlichkeit ihres Lebens und ihrer Lebenswelten maßgeblich beeinflusst. In der wissenschaftlichen Diskussion des Begriffs Empowerment hat dessen Mehrdimensionalität zu unterschiedlichen Schwerpunkten und Teilkonzepten geführt: Empowerment wird dabei als Prozess (empowering) und als Ergebnis (empowered) betrachtet [4-6]. Gleichzeitig werden verschiedene Ebenen von Empowerment unterschieden: individual, organizational und community empowerment [5-9].

Zimmerman [5] differenziert Empowerment-Prozesse und -Ergebnisse auf allen drei Ebenen. Auf individueller Ebene beziehen sich Empowerment-Prozesse auf Erfahrungen, die es Menschen ermöglichen, an Entscheidungsfindungs- und Problemlösungsprozessen teilzuhaben. Sie werden über die Beteiligung an organisatorischem Handeln erreicht. Eine „empowered person“ hat die Gewissheit, in verschiedenen Situationen selbst die Kontrolle übernehmen zu können (intrapersonal component), verfügt über ein kritisches Bewusstsein ihrer Umgebung gegenüber (interpersonal component) und besitzt die nötigen Verhaltensweisen, um die notwendige Kontrolle ausüben zu können (behavioral component). Auf der Ebene des „organizational empowerment“ halten „empowering organizations“ Lernmöglichkeiten für Individuen bereit, die Kontrolle in bestimmten Situationen selbst übernehmen zu können. „Empowered organizations“ durchlaufen Entwicklungsprozesse erfolgreich und beeinflussen politische Entscheidungen. Empowerment-Prozesse auf der Ebene des „community empowerment“ beinhalten den für alle Bürger möglichen Zugang zu Ressourcen (z.B. zu Freizeitanlagen, Präventions- und Gesundheitsförderungsmaßnahmen) und offene Regierungsstrukturen, die die Belange der Bürger ernst nehmen und berücksichtigen. Eine „empowered community“ ist charakterisiert durch Bürgerbeteiligung und durch Interesse an Veränderungsprozessen [5].

\section{Die Umsetzung des Empowerment-Konzepts im BIG-Projekt}

In der vorliegenden Studie werden alle drei EmpowermentEbenen in der Evaluation berücksichtigt: Es werden zum einen Empowerment-Wirkungen bei den Frauen auf individueller Ebe- 


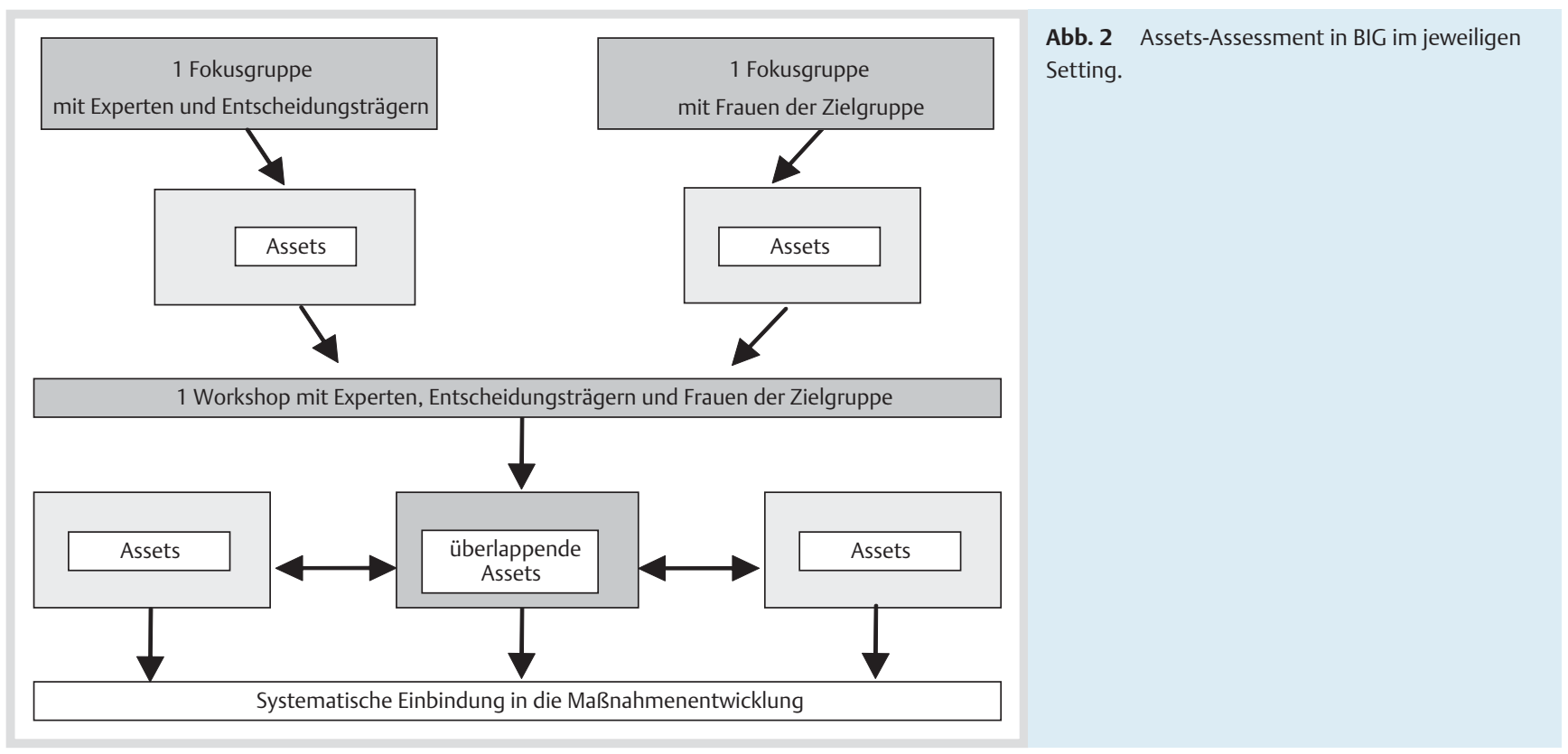

ne untersucht, zum anderen werden Empowement-Prozesse in Bezug auf die beteiligten Frauen auf organisatorischer und gemeindebezogener Ebene betrachtet. Darüber hinaus wird diskutiert, inwiefern diese Prozesse die Entwicklung von individuellen Empowerment-Effekten unterstützen. Im BIG-Projekt wird damit die Ganzheitlichkeit des Empowerment-Konzepts betont, d.h. die Interdependenz der Komponenten Befähigung (Entwicklung individueller Kompetenzen und sozialen Handelns) und Bemächtigung (Erhöhung der Kontrolle über die Lebenswelt und die sie beeinflussende Politik).

\section{Die Anwendung des Empowerment-Konzepts in der BIG Assessment-Phase}

In der initialen Projektphase - der Assessment-Phase - wurde der WHO Assets-Ansatz [10] angewendet, um Assets ${ }^{2}$ („Aktivposten“) für die Förderung körperlicher Aktivität auf individueller, organisatorischer und infrastruktureller Ebene aus Sicht von Frauen aus der BIG-Zielgruppe sowie von BIG-Experten und Entscheidungsträgern zu ermitteln. Zu diesem Zweck wurden jeweils separate Fokusgruppen durchgeführt: in jedem der drei Settings mit Frauen der BIG-Zielgruppe und mit Experten und Entscheidungsträgern ( $\bullet$ Abb. 2).

Insgesamt nahmen 43 Frauen an diesen Fokusgruppen teil und generierten dabei jeweils Assets für die Förderung von Sport und Bewegung auf allen der drei genannten Ebenen. Am AssetsAssessment beteiligten sich Frauen mit unterschiedlichem Migrationshintergrund (z.B. deutsch-russisch, südamerikanisch oder türkisch) sowie allein erziehende und/oder arbeitslose Frauen mit deutschem Hintergrund. Rekrutiert wurden die Frauen in zwei Schritten: Zunächst wurde der Kontakt über verschiedene Experten in den jeweiligen Settings hergestellt, so z.B. über einen Pfarrer, einen Sozialarbeiter oder eine Kindergartenleiterin. Im Anschluss wurden die auf diese Weise gewonnenen Frauen interviewt, um den Kontakt zu weiteren Frauen zu entwickeln.

\footnotetext{
${ }^{2}$ Als Assets oder auch „Aktivposten“ für Bewegung kann jeder Faktor (oder jede Ressource) bezeichnet werden, der Möglichkeiten für Individuen eröffnet, Bewegungsaktivitäten auszuüben. Diese Aktivposten können auf der individuellen, der organisatorischen sowie der infrastrukturellen Ebene vorliegen (vgl. Morgan, et al. 2004)
}

Im Anschluss an die Fokusgruppen erfolgte in jedem Setting ein gemeinsamer Workshop für Experten/Entscheidungsträger sowie die Frauen der Zielgruppe mit der Maßgabe, die jeweils generierten Assets - auch settingübergreifend - vorzustellen und settingbezogen die „überlappenden“ Assets von Experten/ Entscheidungsträgern und den Frauen der Zielgruppe zu identifizieren. Als gemeinsame Assets wurden beispielsweise auf individueller Ebene eine weibliche Übungsleiterin und ein Sozialarbeiter im Wohnquartier genannt, auf organisatorischer Ebene Organisationen wie eine protestantische Kirche und ein Kindergarten und auf infrastruktureller Ebene ein Raum in der lokalen Moschee und Räumlichkeiten eines Sportvereins [11]. Die Ergebnisse des Assets-Assessments wurden systematisch für die Maßnahmenentwicklung in der BIG-Projektplanung berücksichtigt.

\section{Empowerment in der BIG-Projektplanung und Implementation}

Der partizipative Ansatz von BIG beinhaltet eine umfassende Beteiligung von Frauen aus der BIG-Zielgruppe sowie von Experten und Entscheidungsträgern an allen Projektphasen, d. h. der Projektplanung, -implementierung und -evaluierung. Kernstück dieses Ansatzes ist das Konzept der „Kooperativen Planung“ [1]. Insgesamt 37 Frauen aus der Zielgruppe nahmen in der Planungsphase an drei Planungsgruppen - eine Gruppe in jedem Setting - und einer Dachgruppe teil. Die Dachgruppe diente der Steuerung der Aktivitäten der Planungsgruppen. Der Planungsablauf folgte einem standardisierten Schema [1] mit insgesamt 5 Sitzungsterminen. In diesem Zeitraum wurden in jeder Gruppe Maßnahmen zur Förderung von Sport und Bewegung entwickelt und ein konkreter Umsetzungsplan erstellt ( $\bullet$ Abb. 3).

Folgende Interventionsmaßnahmen, die insbesondere von den beteiligten Frauen aktiv in die Diskussion eingebracht wurden, ergaben sich aus diesem Planungsprozess: Wohnortnahe und kostengünstige Bewegungsprogramme mit Kinderbetreuung, Frauenbadezeit, Bereitstellung eines Multifunktionsraums für Treffen der Frauen, Zugang zu Fitnessstudios, Gesundheitsseminare und Projektbüros. Die Projektbüros, die von den Frauen selbst gemanagt wurden, dienten der Organisation und Steuerung der Interventionsmaßnahmen während der Umsetzungs- 


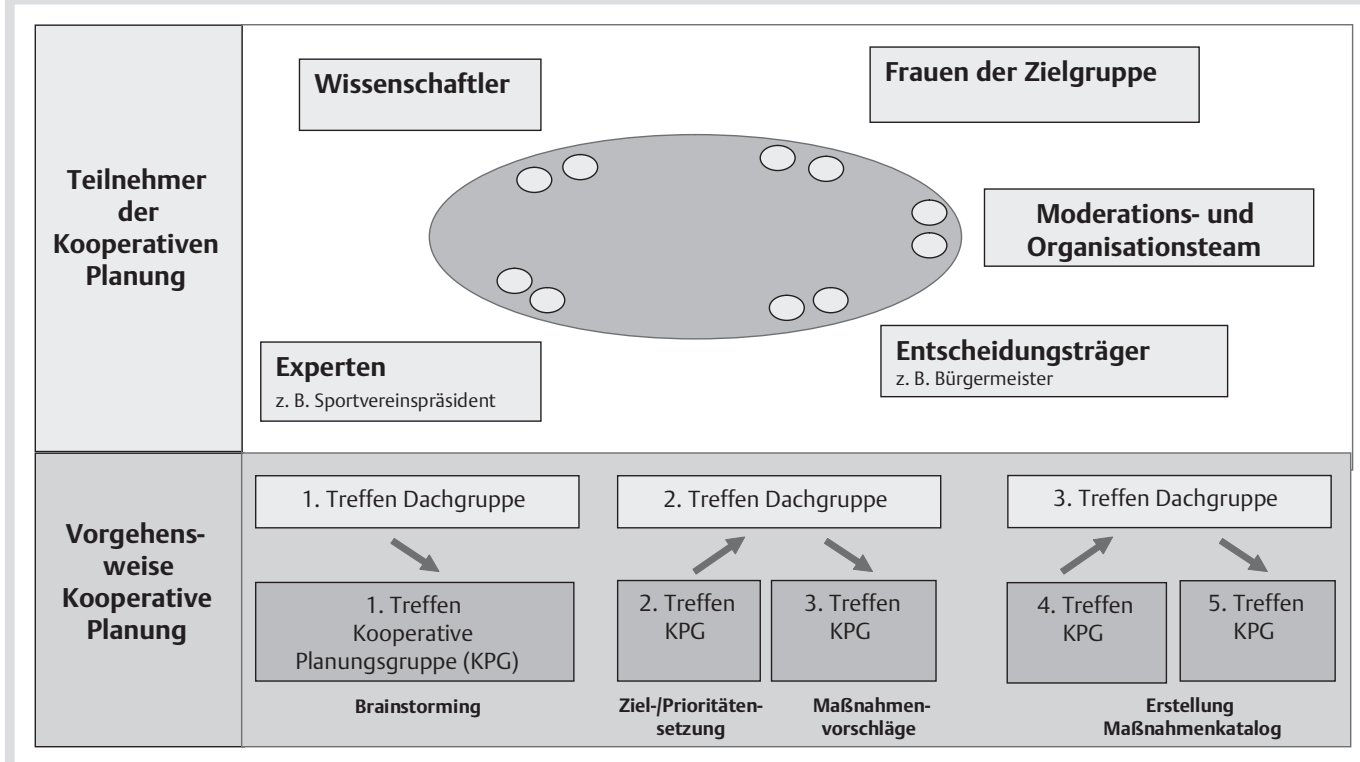

Abb. 3 Teilnehmer und Ablauf der Kooperativen Planung.

phase des Projekts. Dabei setzten sich die Frauen eigenständig für die Umsetzung der von ihnen gewünschten Maßnahmen ein. So konnten sie z. B. die Rektorin einer Schule an ihrem Wohnort überzeugen, ihnen die schuleigene Turnhalle für das BIG-Bewegungsprogramm zur Verfügung zu stellen.

\section{Empowerment in BIG - Ergebnisse der Projektevaluation \\ $\nabla$}

Der Beteiligungsansatz mit dem Ziel des Empowerment fand auch in der Projektevaluation Anwendung. In diesem Kontext wurden die Frauen der Zielgruppe nicht nur im Sinne partizipatorischer Evaluation - z. B. in der Dachgruppe - zur eigenen Analyse und Bewertung der verschiedenen Prozesse und Ergebnisse von BIG aufgefordert. Vielmehr bekamen sie auch Gelegenheit, an der Durchführung der wissenschaftlichen Evaluationsstudien als Mitarbeiterinnen mitzuwirken. Dabei zeigte ein zusätzlich durchgeführter Methodenvergleich, dass die Qualität der Datenerhebung durch die Frauen im Rahmen einer Befragung in den Settings hinsichtlich Rücklaufquote und Erreichung der Zielgruppe einem Standardansatz der Umfrageforschung (Telefonbefragung mit geschulten Interviewerinnen) eindeutig überlegen war.

\section{Datenerhebungsmethodik}

Zur Erhebung der Daten bzgl. der Empowerment-Ergebnisse bei den an BIG beteiligten Frauen wurden halbstrukturierte Interviews mit den Frauen durchgeführt. Auf der Basis eines Interviewleitfadens wurden Daten hinsichtlich durch BIG erfahrener individueller Veränderungen, organisatorischer und gemeindebezogener Beteiligung sowie der Interaktion mit anderen BIGBeteiligten erfragt. Insgesamt wurden 15 Frauen interviewt. In jedem Setting wurden zwei Planungsgruppenteilnehmerinnen, eine langfristige Teilnehmerin an BIG-Bewegungsprogrammen und eine Aussteigerin aus den BIG-Bewegungsprogrammen befragt. Zudem wurden drei Teilnehmerinnen an den BIGSchwimmkursen und der Frauenbadezeit befragt.

Die Interviews wurden auf Tonband aufgenommen und transkribiert. Analysiert wurden die Interviews mithilfe der Qualita- tiven Inhaltsanalyse von Mayring [12], der fünf Analyseschritte vorschlägt: (1) Entscheidung über das einzubeziehende Datenmaterial und die Forschungsfrage, (2) Entscheidung über die Analysetechnik (z.B. Zusammenfassung oder Strukturierung) und den Prozess der Datenanalyse, (3) Definition des Kategoriensystems und der Analyseeinheiten, (4) Überprüfung des Kategoriensystems und (5) Interpretation der Ergebnisse.

Alle 15 Interviews wurden in die Analyse einbezogen. Die Forschungsfrage bezog sich auf individuelle, organisatorische und gemeindebezogene Empowerment-Effekte, die die Frauen durch BIG erfahren haben. Als Analysetechnik wurde die Zusammenfassung gewählt, um das Material auf die wichtigsten Inhalte zu reduzieren. Das Kategoriensystem wurde auf der Basis der Kategorien von Zimmerman [5] entwickelt und beinhaltete Kategorien zum individuellen, organisatorischen und gemeindebezogenen Empowerment. Das Kategoriensystem wurde von zwei unabhängigen Personen überprüft; die Ergebnisse wurden diskutiert und Inkonsistenzen überarbeitet. Beim Materialdurchlauf wurden die Interviewpassagen zu den einzelnen Kategorien zugeordnet und zusammengefasst.

\section{Ergebnisse zum individuellen Empowerment}

In Anlehnung an das theoretische Modell von Zimmerman [5] wurden drei Komponenten des individuellen Empowerment unterschieden: intraindividuelle, interindividuelle und VerhaltensKomponente.

\section{Intraindividuelle Komponente}

Nach den Ergebnissen der Interviews hat sich die Teilhabe an BIG auf die Selbstwirksamkeit der Frauen ausgewirkt. Projektbüroleiterinnen und Teilnehmerinnen an der Kooperativen Planung von BIG trauen sich zu, ihre Wünsche zu äußern und eine Rede vor einer Gruppe von Menschen zu halten, selbst wenn Personen mit bedeutsamen Positionen - wie z.B. der Bürgermeister - im Plenum sitzen. Dabei berichten Frauen mit Migrationshintergrund, dass sie sich jetzt zutrauen, vor Deutschen zu sprechen. Eine Frau berichtet, dass sie sich seit der Mitarbeit an BIG dazu in der Lage fühlt, selbständig, ohne ihren Mann, Ämtergänge zu erledigen. 
„Wie gesagt, früher konnte ich nicht unter diesen Deutschen hereinkommen, und jetzt ist es total anders geworden. Jetzt kann ich halt 'rein, wenn ich Sachen im Rathaus habe; dann ist immer mein Mann gegangen. Ich bin fast nie 'rein, aber seitdem gehe ich immer 'rein, und ich bin halt mehr unter deutschen Leuten. Also ich habe jetzt keine Angst. Früher hatte ich wirklich Angst gehabt, ich wusste nicht, was ich sagen sollte, war sehr skeptisch und habe sie auf Abstand gehalten, aber jetzt ist es nicht mehr so" (Interview 2).

Bei Frauen, die nur an den Bewegungsprogrammen teilgenommen haben, beziehen sich Selbstwirksamkeitseffekte insbesondere auf das Sporttreiben selbst. Diese Frauen berichten, in BIG davon erfahren zu haben, Sport auf eigenem Niveau treiben zu können, und dass Sporttreiben darüber hinaus - im Gegensatz zu den Erfahrungen aus dem Schulsport - Spaß machen kann.

„Z.B. hätte ich früher überhaupt keinen Sport getrieben. Null, also null Sport und keine Bewegung, nichts, gar nichts. Jetzt z.B. fahre ich Fahrrad oder ich laufe, wenn ich irgendwo hin muss, sage ich: du kannst jetzt ja auch da hinlaufen, und ja. Oder also ich bin jetzt nicht mehr so abgeneigt, sage ich mal, zum Sport. (...) Also ich hatte eine Lehrerin, die habe ich gar nicht gemocht, und ja, z.B. ist man dann in der Hitze im Sommer, und unter der Hitze im schönen Wetter ist man da gelaufen, und das war halt nichts für mich mit Kopftuch, und ja, das war nicht schön. Und dann gab es noch Noten, dann ist man halt unter Druck, dieses Unter-Druck-Gesetztsein, das hat mich schon immer fertig gemacht in der Schule“. (Interview 4).

\section{Interindividuelle Komponente}

Die Projektbüroleiterinnen und Teilnehmerinnen an der Kooperativen Planung von BIG entwickelten im Laufe des Projekts ein kritisches Bewusstsein für ihre Lebenswelt. Unter anderem, gewannen die Frauen Verständnis für wichtige Personen, die diese Lebenswelt entscheidend mitbestimmen und beeinflussen. So entwickelten Projektbüroleiterinnen ein Verständnis für verschiedene kulturelle Hintergründe und damit beispielsweise verbundene Barrieren der Sportteilnahme. Hier ist z. B. bei muslimischen Frauen die Zeit des Ramadan als Hinderungsgrund für die Teilnahme an Schwimmbadangeboten zu nennen.

„Ja, die Frauenbadezeit ist am Sonntag losgegangen, da war keine Frau da. Und also die [Name einer Frau] hat auch gesagt, also des liegt wahrscheinlich daran, dass die Frauen nicht mal Wasser in den Mund bekommen dürfen [im Ramadan], nicht mal Schwimmbadwasser, also das war mir auch nicht so klar, dass das wirklich dann auch ein Grund ist. Und die Gefahr, dass man beim Schwimmen natürlich Wasser in den Mund bekommt, noch dazu wenn man nicht schwimmen kann, ist sehr groß“. (Interview 1).

Projektbüroleiterinnen entwickelten zudem gleichermaßen Verständnis für wichtige Entscheidungsträger in Zusammenhang mit der Förderung von Sport und Bewegung in ihrem jeweiligen Setting sowie für Einflussfaktoren, wie z.B. Schwierigkeiten der Erreichbarkeit, die es bei der Abstimmung zu berücksichtigen galt.

„Mit denen, die mitgeholfen haben, also halt über das BIGProjektbüro, bin ich schon in, mit ja so im Sportamt, dann mit der VHS, mit der [Name einer Entscheidungsträgerin], die habe ich jetzt immer noch nicht erreicht. Schon vor ein paar Monaten, oder vor der Sommerpause, das war einfach ganz schwer, da bin ich dran“ (Interview 1).

\section{Verhaltens-Komponente}

Alle befragten Frauen (Projektbüroleiterinnen, Teilnehmerinnen an der Kooperativen Planung und Teilnehmerinnen an den Sport- und Bewegungsprogrammen) berichten über soziale Kontakte, die sie über BIG geknüpft haben. Projektbüroleiterinnen und Kooperative Planungsmitglieder entwickelten soziale Kontakte zu wichtigen Entscheidungsträgern und zu Übungsleitern für Sport und Bewegung. Alle Frauen entwickelten soziale Kontakte untereinander zu Frauen mit und ohne Migrationshintergrund.

„Ja, Veränderungen, ich weiß nicht. Aber ich habe jetzt sehr viele Leute kennen gelernt. Das ist sehr wichtig für mich, weil ja die Umgebung ist ganz anders geworden, und ich habe das ja auch vorhin gesagt, ich bin jetzt ganz anders geworden. Wie sagt man das? Nicht aktiver, sondern ich kann z.B. das sagen, ich komme jetzt halt viel leichter unter deutsche Leute, da habe ich jetzt nicht so viele Schwierigkeiten wie vorher. Da habe ich ein bisschen Veränderung bei mir“. (Interview 2).

\section{Ergebnisse zur Unterstützung des individuellen}

Empowerments durch organisatorische und gemeindebezogene Empowerment-Prozesse

Obwohl die Analyse der organisatorischen und gemeindebezogenen Empowerment-Prozesse primär einer weitergehenden Analyse vorbehalten bleibt, die sich auf Experteninterviews und Dokumentenanalysen stützt, wurden solche EmpowermentProzesse auch in den Interviews z.B. mit den Projektbüroleiterinnen und Teilnehmerinnen an der Kooperativen Planung berichtet. So wurden die Projektbüroleiterinnen beispielsweise in Entscheidungsfindungsprozesse bezüglich der BIG-Bewegungsprogramme in der Volkshochschule und im städtischen Schwimmbad konkret einbezogen.

„Ja, also mit der Frau [Name leitende Mitarbeiterin der VHS] komme ich gut klar. Wir sprechen uns auch ab und das läuft gut soweit. Ich bin dann auch bei ihr im Büro, und wir bestimmen dann auch zusammen, wie wir das machen können. Die [Name einer muslimischen Frau] ist dann auch dabei, auch mit dem Herrn [leitender Mitarbeiter im städtischen Schwimmbad], also das läuft sehr gut, auch mit dem Sportamt" (Interview 1).

Über das BIG-Projekt erhielten alle beteiligten Frauen Zugang zu kommunalen Ressourcen wie Schulturnhallen, Gymnastikräumen und Schwimmbädern. Außerdem öffneten sich die kommunalpolitischen Strukturen für die Belange von Frauen in schwierigen Lebenslagen. In diesem Zusammenhang wurde eine der befragten Frauen von einer Stadträtin angesprochen, ob sie für den Stadtrat kandidieren möchte. Dieser Kontakt zur Stadträtin hatte sich über BIG ergeben. Eine andere Frau wurde angesprochen, das BIG-Projekt bei einem städtischen Gremium zu präsentieren - also als Vorbereitung für eine wichtige Entscheidung über das BIG-Projekt im Stadtrat.

\section{Diskussion \\ $\nabla$}

In der vorgestellten Untersuchung wurde analysiert, welche Wirkungen die umfassende Beteiligung von Frauen in schwierigen Lebenslagen an Planung, Implementierung und Evaluation von Maßnahmen zur Bewegungsförderung auf das Empowerment dieser Frauen hat.

Auf individueller Ebene wurden Empowerment-Wirkungen bei allen drei Befragungsgruppen - Teilnehmerinnen an den Sport- 
und Bewegungsprogrammen, Kooperative Planungsteilnehmerinnen und BIG-Projektbüroleiterinnen - ermittelt. Alle Frauen berichten von Veränderungen ihrer Selbstkompetenz (intraindividuelle Komponente). Allerdings greifen diese Effekte nur bei den Teilnehmerinnen der Kooperativen Planung und den Projektbüroleiterinnen über das Sporttreiben hinaus auf andere Lebensbereiche über, wie z.B. den Erwerb von Kompetenzen im Umgang mit Ämtern. Während alle Befragungspersonen von neuen Sozialkontakten über die Teilnahme an BIG berichten (Verhaltens-Komponente), entstand nur bei den Beteiligten an der Kooperativen Planung und bei den Projektbüroleiterinnen ein Verständnis für wichtige Personen und Entscheidungsträger (interindividuelle Komponente). Empowerment-Prozesse im Sinne der Bemächtigung von Frauen in schwierigen Lebenslagen auf organisatorischer und gemeindebezogener Ebene, wurden fast ausschließlich von den Teilnehmerinnen der Kooperativen Planung und den Projektbüroleiterinnen berichtet. Beispielsweise wurden Projektbüroleiterinnen in Entscheidungen von Organisationen wie der VHS einbezogen und sogar in einem Fall für eine Stadtratskandidatur gewonnen. Dies lässt vermuten, dass solche Bemächtigungs-Prozesse, die über die verstärkte Partizipation der Frauen an der Planung, Implementierung und Evaluation von Gesundheitsförderungsmaßnahmen entstehen, die Entwicklung von eher generellen, über Sport und Bewegung hinaus gehenden Empowerment-Effekte unterstützen.

Kritisch ist anzumerken, dass die Ergebnisse der Untersuchung eher explorativen Charakter haben. Weiterhin beziehen sich die Ergebnisse der Studie nur auf Aussagen der Frauen, während Aussagen anderer Personen - wie z. B. an BIG beteiligte Experten und Entscheidungsträger - nicht in diese Ergebnisdarstellung einbezogen wurden. Außerdem wurden die Interviews nur exemplarisch mit 15 an BIG beteiligten Frauen geführt und stellen somit keine Vollerhebung dar.

Nichtsdestoweniger ist die vorliegende Untersuchung eine der wenigen, die das von verschiedenen Autoren geforderte „Mehrebenen-Konzept“ $[5,7,8]$ zugrunde legt, um EmpowermentWirkungen eines partizipativen Gesundheitsförderungsprogramms zu analysieren [6]. Die Ergebnisse deuten an, dass Frauen in schwierigen Lebenslagen durch eine umfassende Beteiligung an allen Phasen eines Gesundheitsförderungsprogramms, d.h. sowohl an Planung, Implementierung wie auch Evaluation, nicht nur auf individueller Ebene befähigt werden, Kompetenzen und soziale Handlungsfähigkeit weit über den Bereich von Sport und Bewegung hinaus zu entwickeln, sondern zudem bemächtigt werden, Einfluss auf ihren Alltag betreffende Organisationen, Lebenswelten und Politiken zu üben und damit letztlich - ganz im Sinne der WHO-Empfehlungen zur Gesundheitsförderung [2,3] - in die Lage versetzt werden, eigenständig die Kontrolle über Determinanten ihrer Gesundheit im Sinne der selbst bestimmten Beeinflussung ihrer persönlichen Lebensführung und ihrer jeweiligen Lebensbedingungen zu übernehmen.

\section{Anmerkungen}

$\nabla$

Das BIG Projekt wurde gefördert vom Bundesministerium für Bildung und Forschung im Rahmen der Präventionsforschung des Bundes (01EL0402).

\section{Literatur}

1 Rütten A. Investition in Gesundheit: Kompendium zur Gesunden Region in Westsachsen. Stuttgart: Nagelschmid; 2000

2 WHO. Ottawa Charter for Health Promotion. First International Conference on Health Promotion. Ottawa, 21. November 1986. WHO/HPR/ HEP/95.1; 1986

3 WHO. Health promotion glossary. Geneva: WHO; 1998

4 Bernstein E, Wallerstein N, Braithwaite R et al. Empowerment Forum: A Dialogue between Guest Editorial Board Members. Health Education Quarterly 1994; 21 (3): 281-294

5 Zimmerman MA. Empowerment Theory. Psychological, Organizational and Community Levels of Analysis. In: Rappaport J, Seidman E, Hrsg. Handbook of Community Psychology. New York: Kluwer Academic; 2000

6 Wallerstein $N$. What is the evidence of effectiveness of empowerment to improve health. WHO, Regional Office for Europe: Copenhagen; 2006

7 Wallerstein N. Powerlessness, empowerment, and health: Implications for health promotion programs. American Journal of Health Promotion 1992; 6 (3): 197-205

8 Israel BA, Checkoway B, Schulz A et al. Health Education and Community Empowerment: Conceptualizing and Measuring Perceptions of Individual, Organizational, and Community Control. Health Education Quarterly 1994; 21 (2): 149-170

9 Zimmerman MA. Psychological Empowerment: Issues and Illustrations. American Journal of Community Psychology 1995; 23 (5): 581-599

10 Morgan A, Ziglio E, Harrison D et al. Assets for Health and Development Programme Overview - Making the Case. WHO - European Office for Health and Development; 2004

11 Ruetten A, Abu-Omar K, Seidenstuecker $S$ et al. Assets Movers for Health - Concept and Use of Assets in Promoting Healthy Behaviour. In: Morgan A, Ziglio E, Hrsg. Health Assets. WHO, [in Druck]

12 Mayring P. Qualitative Inhaltsanalyse. Grundlagen und Techniken. 9. Aufl. Weinheim: Beltz; 2007 